\title{
Cellular probes on the move
}

\section{Sanford M. Simon}

Over the past 50 years, many of the achievements in biochemistry and cell biology have been due to a reductionist-"grind and find"-approach. Step by step, organelles and biochemical pathways were separated and characterized and the relevant proteins were purified, cloned, and sequenced. The in vitro reconstitution of enzymatic pathways and membrane transport processes validated this reductionism. However, as the article by Yokoe and Meyer on pp. 1252-1256 of this issue illustrates, a new paradigm is rapidly spreading in cell biology: The intact living cell as a biological reaction chamber.

It has always been appreciated that the cell is not merely a homogenized mix. Subcellular compartmentalization is essential for many cellular functions such as glycosylation (in the secretory pathway), energetics (in the mitochondria and chloroplast), digestion (in the lysosome), and neurotransmitter release (at the synaptic junction). Each of their many partial reactions is important, but understanding their consequences for the cell requires knowledge of the spatial and temporal organization. For example, Hodgkin and Huxley demonstrated that the electrical excitability underlying the action potential could be understood from a patch of membrane. In contrast, understanding the integration of information in a cerebellar Purkinje cell requires detailed knowledge of the spatial distribution of membranes and ion channels and as well as the temporal history of their activity. Thus, for cell physiology, the intact cell always remained a method of choice.

Similarly, understanding many cell biological processes requires a detailed spatial and temporal knowledge of proteins, nucleotides, ions (especially calcium and protons) and other signaling molecules in the living cell. Many scientists have been developing the technology to permit spatial and temporal analysis of these molecules (perhaps driven by the knowledge that soon every gene in the genome will be purified, cloned, sequenced, and expressed). There have been two major technological advances. First, there have been improvements in instrumentation. This includes improvements in optics, light-sensitive cameras and photomultiplier tubes, and the ongoing refinements in reflectance and fluorescence confocal microscopy, which allows optical

Sanford M. Simon is at the Laboratory of Cellular Biophysics, Box 304, The Rockefeller University, 1230 York Avenue, New York, NY (simon@rockvax.rockefeller.edu). sectioning of living cells. The second advance is the development of fluorescent probes that can be used in living cells to quantitatively assay the spatial and temporal behavior of intracellular molecules.

One use of fluorescent probes is for the study of intracellular signaling molecules. A bioluminescent protein, aequorin ${ }^{1}$, was first used over 25 years ago to demonstrate the existence of transient localized changes of calcium in muscle and in the presynaptic terminal of neurons during an action potential. Aequorin had limitations. It could only be used to qualitatively assay calcium changes and only with cells large enough to allow microinjection. However, in recent years a series of dyes has been developed that surmounts these problems: They can be calibrated and loaded into almost any cell type $e^{2}$. These dyes were initially developed to measure the intracellular signaling molecules calcium, protons $(\mathrm{pH})$ and adenosine $3^{\prime}, 5^{\prime}-$ cyclic monophosphate. Additionally, probes have been developed that can transiently release these molecules, thereby allowing experimental control over their subcellular distribution of these molecules ${ }^{2,3}$. This technology has been expanded to include fluorescent sensors for proteins such as calmodulin, profilin and myosin II regulatory light chain phosphorylation".

A second group of probes was used to study of transport properties with the cell. Probes were introduced into the endocytic pathway by conjugation to lectins, such as concanavalin A, or cellular ligands, such as nerve growth factor ${ }^{5-7}$. The use of fluorescent probes that were, in turn, sensitive to protons allowed for $\mathrm{pH}$ measurements at steps along the endocytic pathway. A powerful tool in the study of these fluorophores was the use of fluorescence recovery after photobleaching, which has been used to measure the mobility of protein and lipids within cellular membranes ${ }^{9}$, intracellular communication ${ }^{10}$, and the rate of diffusion into nuclei ${ }^{11}$.

Recently, probes have been used to mark by fluorescence proteins in living cells. Green fluorescent protein (GFP), from the jellyfish Aequorea vitoria, is naturally fluorescent. GFP was first introduced in the nematode as a marker for gene expression ${ }^{12}$. When expressed by itself, GFP fluoresces diffusely within the cell, thus functioning as a reporter for protein localization within cells. In the past two years, many studies have have focused on either optimizing the fluorescent properties of GFP or using it to study the location and movement of particular GFP-fusion proteins in living cells. In their article, Yokoe and Meyer modify the technique by using a spatially focused UV-laser pulse to enhance the fluorescence of GFP. This allows them to measure the diffusion of K-ras and characterize its dynamic equilibrium between a cytosolic and membrane-bound form.

One important challenge for the future is to assay the enzymatic activity of proteins in living cells. A protein's location within a cell may not always be coincident with its activity. Activation or inhibition of a protein may depend upon a second agent. However, several studies suggest that even this challenge may soon be surmounted. One of the early measurements of the spatial compartmentalization of enzymatic activities in cells was the use of substrates to mark the lumen of the Golgi for electron microscopy. Similarly, its activities in lipid biogenesis have been used to mark it for light microscopy ${ }^{13}$. Recently, it has been demonstrated that the use of fluorescent antibodies and substrates allows the subcellular distribution and activity of prostaglandin endoperoxide synthase- 1 and 2 to be simultaneously measured in the same cell $^{14}$. Further advances require technology that can simultaneously resolve and quantify multiple signals ${ }^{15}$. At the Microscopy Society of America's annual meeting in August (Minneapolis, MN), a microscope was demonstrated with five, simultaneous, photomultiplier detectors operating in conjunction with four, individually controlled, laser lines. Thus, for the moment, the limiting factor may be the development of probes with sufficiently distinct fluorescent lines to allow the simultaneous measurement of different cellular activities.

1. Shimomura, O. and Johnson, F.H. 1969. Biochemistry 8:3991-3997.

2. Tsien, R.Y. 1992. Am. J. Physiol 263:C723-C728.

3. Kao, J.P.Y., Harootunian, A.T., and Tsien, R.Y. 1989. J. Biol. Chem. 264:8179-8184.

4. Giuliano, K.A., Post, P.L., Hahn, K.M., and Taylor, D.L. 1995. Annu. Rev. Biophys Biomol. Struct. 24:405-434.

5. Nossal, G.J. and Layton, J.E. 1976. J. Exp. Med. 143:511-528.

6. Storrie, B. and Edelson, P.J. Cell 11:707-717.

7. Levi, A., Shechter, Y., Neufeld, E.J., and Schlessinger, J. 1980. Proc. Natl. Acad. Sci. USA 77:3469-3473.

8. Tycko, B. and Maxfield, F.R. 1982. Cell 28:643-651.

9. Edidin M. and Fambrough, D. 1973. J. Cell Biol. 57:27-37.

10. Wade, M.H., Trosko, J.E., and Schindler, M. 1986. Science 232:525-528.

11. Peters, R. 1983. J. Biol. Chem. 258:11427-11429.

12. Chalfie, M., Tu, Y., Euskirchen, G., Ward, W.W., and Prasher, DC 1994. Science 263:802-805.

13. Lipsky, N.G. and Pagano, R.E. 1985. Science 228:745-747.

14. Morita, I., Smith, W.L., DeWitt, D.L., and Schindler, M. 1995. Biochemistry 34:7194-7199.

15. DeBiasio, R., Bright, G.R., Ernst, L.A., Waggoner, A.S., and Taylor, D.L. 1987. J. Cell Biol. 105:1613-1622. 